\title{
The effect of personal continuity of care on evidence-based management of patients with asthma in primary care
}

K Khunti

SUMmari

Continuity of care is much valued by doctors and patients, but little is known about the effect of personal continuity of care on the clinical management of patients. This pilot study examined the effect of continuity of care on the quality of care of patients with asthma in one general practice.

\section{INTRODUCTION}

Patients may consult many different doctors in a group practice. ${ }^{1}$ However, continuity of care, defined in general practice as care from one doctor usually spanning an extended time and more than one episode of illness ${ }^{2}$ is much valued by doctors. There is also evidence that satisfaction of patients and staff is generally associated with high levels of personal continuity of care. ${ }^{3}$ A lack of continuity of care is associated with some additional morbidity, an increased number of relationship problems, 'difficult' consultations and non-attendances, and an increase in the use of open access clinics. ${ }^{4}$ However, little is known about the effect of personal continuity of care on the clinical management of patients. The hypothesis of this pilot study was that increased continuity of care of patients would lead to improved quality of care of patients with asthma.

\section{MethoD}

The study was conducted in an inner city training practice with six principals with a list size of 9800 patients with $14 \%$ of patients being over the age of 65 years. We have two practice nurses who are trained to run the asthma clinic. The notes of patients were retrospectively reviewed to establish the impact of personal continuity of care on clinical management of patients with asthma. A continuity score was obtained from each patient, this being the number of consultations out of the past twelve with the doctor they consulted most frequently. Therefore, they may have been registered with a different doctor. Expressed as a percentage, this is the usual prime of continuity index. ${ }^{5}$ The quality of care of patients with asthma were based on variables derived from national evidence-based audit standards. ${ }^{6}$ Sample size was derived from recommendations in a national audit protocol using Epi Info. Two hundred and fifty notes were selected by random numbers out of 708 patients with asthma on the computer disease register. Analysis was only carried out for five doctors as one doctor had only recently joined the practice.

\section{RESULTS}

One hundred and twenty six patients were male (50.4\%). Table 1 shows the results of the effect of continuity of care on patients with asthma. Doctors A to D are all full time principals and Doctor $\mathrm{E}$ works half time in the practice. The variation in continuity of care between the five doctors was highly significant. Dr D has the highest continuity score and the highest compliance with four out of the five variables of asthma management. However, Dr A with a high continuity score has the lowest compliance with four out of the five variables.

\section{CONCLUSIONS}

I believe this is the first study to report on the impact of continuity of care on a chronic disease in general practice. However, one must be cautious when making conclusions from this pilot study which is based on patients of one practice. There are many confounding factors in this study which only measured the process of care given to patients with asthma and did not address the impact of continuity of care on outcomes. A major confounder is that although asthma is primarily managed by practice nurses, it is up to the practitioners to refer their patients with asthma to the asthma clinic. However, this study did not determine the proportion of patients referred to the asthma clinic by the five doctors.

\section{The recording of evidence-based variables varied} enormously between the practice doctors. However, it was better than those reported in a study of inner city practices in east London which used similar criteria. ${ }^{7}$ Although increased personal continuity of care is associated with high levels of patient satisfaction, this study shows that it may not be related to improved clinical care. Correlation was not conducted because of a small sample of only five doctors. The quality of care given to patients with asthma also differed between the various performance measures and between the individual general practitioners.

\section{Kamlesh Khunti \\ Clinical Lecturer \\ Eli Lilly National Clinical \\ Audit Centre, Department of \\ General Practice and \\ Primary Health Care, \\ Leicester General Hospital, \\ Gwendolen Road, Leicester LE5 $4 P W$. \\ Date received: 12/12/97 \\ Date accepted: 09/03/98}

Asthma in Gen Pract 1998 6(1): $12-13$.

Table 1: Relationship between the number (\%) of patients with variables recorded annually compared with the continuity score $(\%)$ for each general practitioner

a 11 patients registerd with $\mathrm{Dr} F$ are excluded from analysis

\begin{tabular}{|c|c|c|c|c|c|c|c|}
\hline Doctor & A & B & C & D & E & & \\
\hline $\begin{array}{l}\text { Number of patients } \\
\text { registered (Apr 1994) }\end{array}$ & 2598 & 2083 & 2091 & 2005 & 751 & & \\
\hline $\begin{array}{l}\text { Number of patients } \\
\text { with asthma (\%) }\end{array}$ & $178 \quad(6.9)$ & $140 \quad(6.7)$ & 151 (7.2) & $136 \quad(6.8)$ & $80(10.7)$ & & \\
\hline $\begin{array}{l}\text { Number of patients } \\
\text { selected for audit }{ }^{\mathrm{a}}(\%)\end{array}$ & $64 \quad(2.5)$ & $55 \quad(2.6)$ & 41 (2.0) & 49 (2.4) & $30 \quad(4.0)$ & & \\
\hline Continuity score (\%) & 52.3 & 44.5 & 38.7 & 56.8 & 33.9 & & $x^{2} 71.8$ df4 $p<0.0001$ \\
\hline Variables: & \multicolumn{7}{|c|}{ Practice average } \\
\hline $\begin{array}{l}\text { Smoking habit } \\
\text { recorded }\end{array}$ & $72(31.2)$ & 15 (23.4) & $17(30.9)$ & $11(26.8)$ & 19 (38.8) & $10(33.3)$ & $x^{2} \quad 3.2 d f 4 p<0.48$ \\
\hline $\begin{array}{l}\text { Inhaler technique } \\
\text { checked }\end{array}$ & $120(50.2)$ & $17(26.6)$ & $28(50.9)$ & $21(51.5)$ & $37(75.5)$ & $17(56.7)$ & $x^{2} 27.4 d f 4 p<0.001$ \\
\hline Prophilaxis & $135(56.5)$ & $45(70.3)$ & $26(47.3)$ & $19(46.3)$ & $32(65.3)$ & $13(43.3)$ & $x^{2} 12.3 d f 4 p<0.015$ \\
\hline Symptoms reviewed & $115(48.1)$ & $19(29.7)$ & $25(45.5)$ & $21(51.2)$ & $34(69.4)$ & $16(53.3)$ & $x^{2} 18.2 d f 4 p<0.001$ \\
\hline Peak flow recorded & $97(40.6)$ & $14(21.9)$ & $22(40.0)$ & $16(39.0)$ & $33(67.3)$ & $12(40.0)$ & $x^{2} 23.9$ df4 $p<0.0001$ \\
\hline
\end{tabular}


Furthermore, the results of this study provide some evidence that no one measure of quality should be used itself to represent different aspects of the quality of primary care. Some health authorities are now producing performance indicators for the general practices they administer. ${ }^{8}$ Therefore, these performance measures must be interpreted appropriately. Further large studies need to be carried out to establish the impact of personal continuity on clinical care of patients with asthma.

\section{References}

1. Freeman G K and Richards F C. How much personal care in full group practices?. BMJ 1990; 301: 1028-30.

2. Freeman G. Priority given by doctors to continuity of care. $J R$ Coll
Gen Pract 1985; 5: 423-6.

3. Hjortdahl $\mathrm{P}$ and Laerun E. Continuity of care in general practice: effect on patients satisfaction. BMJ 1992; 304: 1287-90.

4. Sweeney K G and Gray D P. Patients who do not receive continuity of care from their general practitioner - are they a vunerable group? $\mathrm{Br} J$ Gen Pract 1995; 45: 133-5.

5. Breslau N and Reeb K G. Continuity of care in the university-based practice. J Med Aduc 1975; 50: 965-9.

6. Lakhani M, Baker R, Khunti K. Monitoring asthma. Eli Lilly

National Clinical Audit Centre. Leicester, University of Leicester, 1994.

7. Feder G, Griffiths C, Highton C et al. Do clinical guidelines

introduced with practice education improve care of asthmatic and

diabetic patients? A randomised controlled trial in general practices in east London. BMJ 1995; 311: 1473-8.

8. Majeed F A and Voss S. Performance indicators for general practice. BMJ 1995; 311: 209-10.

\title{
Myopia in an atopic patient
}

\author{
A Sheikh
}

\section{CASE REPORT}

A 21 year old student presented to his GP in 1991 with a three year history of increasing blurring of vision. During this time he had been repeatedly assessed by his optician who had diagnosed myopia and astigmatism. Glasses and contact lenses of increasing strengths had been prescribed with little benefit. Following the last visit the optician had written to his GP advising referral to an ophthalmologist for further assessment. A review of his history revealed that he was atopic suffering from asthma, eczema, allergic conjunctivitis and perennial rhinitis. There was no other history of note and no family history of eye disorders.

Assessment at the local eye hospital confirmed the profound myopia with a visual acuity of $6 / 36$ and $6 / 24$ in the right and left respectively. Keratoscopy revealed the findings of corneal irregularity and thinning characteristic of keratoconus. Rigid gaspermeable contact lenses were prescribed and a corrected visual acuity of $6 / 9$ and $6 / 6$ was achieved.

\section{Discussion}

Keratoconus was first described in $1854 .{ }^{1}$ It is a relatively common degenerative disease of the cornea with a prevalence of approximately 1 in $2000 .^{2}$ The condition affects all races with a slight preponderance for females. The pathological defect lies in Bowman's layer of the cornea; typical findings include keratocyte degeneration and disruption.

In approximately $6-8 \%$ of cases there is a positive family history; both autosomal dominant and recessive patterns of inheritance have been identified. ${ }^{3}$ For the others, no specific cause has yet been identified. Associations have been noted with a number of systemic and ocular disorders including Down's syndrome, Marfan's syndrome and retinitis pigmentosa. The most frequently described association, however, is with atopic conditions such as asthma, eczema and hay fever.
The evidence in support of an association between keratoconus and atopy is reasonably strong. Since the initial reported association in the early $1930 \mathrm{~s},{ }^{4} \mathrm{a}$ number of case reports have appeared in the literature describing the coexistence of atopic conditions and keratoconus..$^{5-7}$ This data has been further supported by descriptive studies that have consistently shown a high prevalence of atopic conditions in keratoconus patients. In a US study of 162 patients with keratoconus an $18 \%$ prevalence of asthma, and $35 \%$ prevalence of hay fever was observed. ${ }^{8}$ A British study of 67 patients revealed a history of atopic disease in $56 \%$ of patients; $28 \%$ of this group had asthma. ${ }^{9}$ More recently, in the largest study of its kind involving 38 centres and 1,579 patients, the Collaborative Longitudinal Evaluation of Keratoconus group reported atopic disease in $34.6 \%$ of patients, with $13 \%$ diagnosed as suffering from asthma. ${ }^{10} \mathrm{In}$ the above cases, the prevalence of asthma and atopy was significantly higher than that of the general population.

Data from controlled studies is less uniform. Lowel et al. in their small study of 31 cases failed to detect any significant difference in atopic traits between keratoconus patients and a similar number of unmatched controls. ${ }^{11}$ Their failure to detect a significant difference may have been due to a lack of power of the study. In a larger study of 182 cases and 100 matched controls, atopy was observed in $35 \%$ compared with $12 \%$ of the controls. The most common allergic disease encountered was hay fever, followed by asthma and eczema. It was also noted that serum $\operatorname{IgE}$ was significantly raised $(\mathrm{p}<0.001)$ in the keratoconus group and markedly so in those cases with associated atopic disease. ${ }^{12}$

Whilst the exact nature of the association between atopy and keratoconus is unclear, the frequency of their co-existence suggest that this is not simply a chance observation. Excessive eye rubbing by atopic patients has been suggested as a possible cause although the evidence in support of this is very

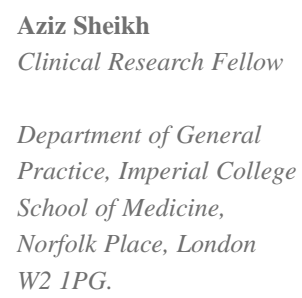

Asthma in Gen Pract 1998; 6(1): 13-14 